\title{
Numerical simulation of blast induced vibration propagation
}

\author{
Duhee Park ${ }^{\text {i) }}$ and Jae-Kwang Ahn ${ }^{\text {ii) }}$ \\ i) Associate professor, Department of Civil and Environmental Engineering, Hanyang University, 506 Jaesung Civil Engr Bldg, \\ Wansimni-ro, Sungdong-gu, Seoul, 133-791, Korea. \\ ii) Ph.D Student, Department of Civil and Environmental Engineering, Hanyang University, Korea..
}

\begin{abstract}
We present a numerical model to simulate blast induced vibration propagation. The model applies normal pressure to the walls of a cavity, which represents the fractured zone formed by the blasting. The material damping is modeled using the Rayleigh damping formulation. The model is verified through comparisons with two sets of analytical solutions. The comparisons demonstrate that the numerical model accurately predicts the attenuation both at near-field and far-field. The near-field attenuation is not greatly influenced by the damping, whereas the far-field vibration is dependent on the material damping. The far-field attenuation also is shown to be sensitive to the frequencies selected for the Rayleigh damping formulation. We propose a procedure to select optimum frequencies for the Rayleigh damping formulation. The proposed numerical model can be used to estimate the dynamic response nearby structures, as well as to develop site-specific source-to-site attenuation relationship.
\end{abstract}

Keywords: blast induced vibration, numerical model, near-field, far-field, rayleigh-damping formulation

\section{INTRODUCTION}

When an explosive detonates, shock waves are generated and propagated from the source. The amplitude of the wave decays in high-intensity with distance from the charge. Blast-induced vibration may have importance influence on adjacent above-ground and underground structures, pipelines, and slopes.

The characteristics of shock wave propagated though geologic media can be estimated from an attenuation relationship or a numerical simulation. In design, the attenuation relationship, which relates the peak particle velocity (PPV) with scaled distance (function of separation distance and charge weight), is most often used to predict the amplitude of vibration (Dowding, 1984). However, because the full range of the attenuation from the source to site is not well defined, the attenuation curves used in practice cannot be used to predict the vibration close to the detonation, or the near-field. Because a typical construction blasting (e.g. tunneling) occurs underground and measurements are made at the surface, the "within media" near-field vibration is even more difficult to predict. A numerical simulation can be used to estimate the near-field vibration. The numerical model can also be used to perform dynamic analysis of structures for detailed assessment of the potential damage.

In this study, we simulate the propagation and attenuation of the blast-induced stress waves through geologic medium from a series of axi-symmetric finite difference (FD) analyses. FD analyses are verified through various forms of analytical solutions. Recommendations on modeling the fracture zone and process for selecting the optimum frequencies for the Rayleigh damping formulation are also outlined.

\section{CLOSED-FORM AND EMPIRICAL ATTENUATION CURVES}

The amplitude of vibration induced by vibration decreases with distance from the source. Decay of vibration is produced by two phenomena, which are geometrical spreading and material damping (Dowding, 1996). The geometrical spreading, also called radiation damping, is caused by the expansion of the surface over which vibration energy is transmitted. The material damping is caused by the nonlinear hysteretic behavior of the geologic media.

The following theoretical equation for geometrical damping calculates the decay of the amplitude of vibration with distance:

$$
A_{R}=A_{a}\left(\frac{a}{R}\right)^{s}
$$

where $A_{a}=$ amplitude of vibration at a distance $a$ from the source, $A_{R}=$ vibration at a distance $R$ from the source, $s=$ geometric damping coefficient. For spherical wave propagation for an underground source, $s=1$. The equation can only be used to calculate the 
decrease in amplitude with distance at far-field, and therefore denoted FA (far-field attenuation equation). The following equation is used to calculate the attenuation caused material damping.

$$
A_{R}=A_{a} e^{-\frac{2 \pi f \xi}{c}(R-a)}
$$

where $c$ is wave velocity of the media, $f$ is the predominant frequency of the motion, and $\xi$ is the small strain damping ratio. The material damping term is denoted as DA (damping attenuation equation) in this paper. DA is multiplied to FA to estimate the decay in the amplitude of vibration due to both geometrical spreading and material damping. The combined equation has been widely used to predict the decay of motion. However, the equation cannot be applied to predict the near-field vibration, which has been reported to be different from far-field attenuation. The equations also only describe the amplitude, and do not provide the equations of the time series of the propagated motion.

Mathematical descriptions of spherically diverging elastic waves instigated by impulse pressure were proposed by Duvall (1953). The functions are based on assumptions that the medium is perfectly elastic with zero damping and that the pressure is applied normal to the spherical surface. Duvall (1953) proposed analytical velocity equations for continuous medium subjected to the following impulse loading:

$$
\begin{aligned}
& p(t)=p_{0}\left(e^{-\alpha t}-e^{-\beta t}\right) \\
& \alpha=\frac{n \omega}{\sqrt{2}}, \quad \beta=\frac{m \omega}{\sqrt{2}}, \omega=\frac{c}{a}\left[\frac{(1-2 v)^{1 / 2}}{(1-v)}\right]
\end{aligned}
$$

where $n, m=$ decay constants, $v=$ Poisson's ratio of rock, $c$ = propagation velocity of longitudinal wave, $a=$ radius of cavity, $\omega=$ angular frequency. The proposed velocity equations is as follows:

$$
\begin{aligned}
V(R, \tau) & =\frac{3 P_{0}}{2 \rho c^{2}}\left(\frac{a}{R}\right) \cdot f(\tau)+\frac{3 P_{0}}{2 \rho c^{2}}\left(\frac{a}{R}\right)^{2} \cdot q(\tau) \\
f(\tau)= & \frac{2 n^{2}}{3} \frac{e^{-n \omega \tau / \sqrt{2}}}{3-2 n+n^{2}}-\frac{2 m^{2}}{3} \frac{e^{-m \omega \tau / \sqrt{2}}}{3-2 m+m^{2}} \\
& -\frac{e^{-\omega \tau / \sqrt{2}}}{\sqrt{2} \sqrt{3-2 n+n^{2}}} \cdot 2 \sin \left(\omega \tau+\theta_{1}-2 \theta_{3}\right) \\
& +\frac{e^{-\omega \tau / \sqrt{2}}}{\sqrt{2} \sqrt{3-2 m+m^{2}}} \cdot 2 \sin \left(\omega \tau+\theta_{2}-2 \theta_{3}\right) \\
q(\tau)= & -n \frac{e^{-n \omega \tau / \sqrt{2}}}{3-2 n+n^{2}}+m \frac{e^{-m \omega \tau / \sqrt{2}}}{3-2 m+m^{2}} \\
+ & \frac{e^{-\omega \tau / \sqrt{2}}}{\sqrt{2} \sqrt{3-2 n+n^{2}}} \cdot \sqrt{3} \sin \left(\omega \tau+\theta_{1}-\theta_{3}\right) \\
& -\frac{e^{-\omega \tau / \sqrt{2}}}{\sqrt{2} \sqrt{3-2 m+m^{2}}} \cdot \sqrt{3} \sin \left(\omega \tau+\theta_{2}-\theta_{3}\right)
\end{aligned}
$$

where, $V=$ velocity, $\quad \rho=$ density of rock,,$R=$ distance from center of cavity, $\tau=$ time ( $\tau=t-(r-a) / c), \theta_{1}, \theta_{2}$, $\theta_{3}=$ phase angle, $\theta_{1}=\tan ^{-1}(\sqrt{2} /(1-n))$, $\theta_{2}=\tan ^{-1}(\sqrt{2} /(1-m)), \quad \theta_{3}=\tan ^{-1} \sqrt{2}$. The particle velocity of spherical propagation has two terms, one which decays as $1 / R$, and the other which decays as $1 / R^{2}$ (Ziolkowski, 1993). Outgoing waves has been considered the function of $f(\tau)$ and $q(\tau)$ that represent the time series and the shape of propagation. The empirical equation is developed for undamped elastic medium with a Poisson's ratio of 0.25 . It is the only theoretical solution can be used for both near-field and far-field propagation. The model is denoted NFA (near-field and far-field attenuation curve) in this paper. It should be noted that the analytical solution represents the "true" solution for undamped medium.

The calculated attenuation curves for undamped case using equations (1), (4) and (6) are compared in Fig. 1. A spherical cavity with a radius $(a)$ of $1 \mathrm{~m}, n=0.25, m$ $=0.5, v=0.25, \rho=2,500 \mathrm{~kg} / \mathrm{m}^{3}, c=2,000 \mathrm{~m} / \mathrm{s}$, and $p_{0}$ $=1 \mathrm{MPa}$ were used for the equation of Duvall (1953). Fig. 1 demonstrates the far-field equations (Eq. (1) and (4)) are identical. However, NFA is shown to be lower than FA. Comparison illustrate that the near-field attenuation is greater than the far-field attenuation and that the attenuation is not linear in a log-log plot.

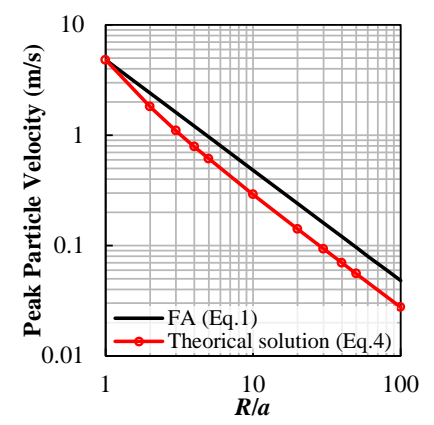

Fig. 1. Comparison of FA and NFA attenuation curves.

The effect of the frequency (duration) of the loading on the calculated attenuation curve is shown in Fig. 2. Three combinations of parameters $n$ and $m$ are used to vary the duration of the loading function, as shown in Fig. 2a. The calculated attenuation curves are shown in Fig. 2b. The slope of the attenuation curves are displayed in Fig. 2c. The attenuation curve is shown to be dependent on the duration, or the frequency, of the impulse loading. Also shown in the Fig. 2c is the slope calculated from FA, Eq (1). It is also demonstrated that the slope converges to unity for all loadings using NFA. The approximate value of $R / a$ at which the slope of the attenuation curve converges is 30 . For FA, the slope is unity for undamped medium and spherical wave propagation. The comparisons highlight that FA and NFA converges at $R / a$ exceeding 30 . The far-field attenuation for undamped medium is independent of the loading frequency, whereas the near-field attenuation is influenced by the frequency of the impulse load. 

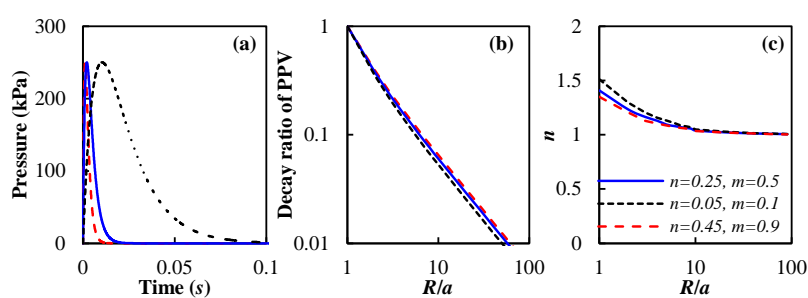

Fig. 2. Influence of impulse function on attenuation curve: (a) impulse function time series (b) attenuation curves (c) slopes of attenuation curves.

\section{NUMERICAL ANALYSIS}

We performed a series of axi-symmetric analyses to simulate the propagation of blast-induced waves through elastic continuum media and to develop representative attenuation curves. Numerical analyses were performed with FLAC ${ }^{2 \mathrm{D}}$ v. 7.0 (Itasca Consulting Group, 2011), a commercial finite difference (FD) analysis program.

The fracture zone caused by blasting is modeled as a spherical cavity. It is assumed that the blasting generates elastic waves that are transmitted to the geologic media from the interface between the fractured zone and the elastic media. The blast load is applied in a normal direction to the cavity surface in the form of either pressure and velocity time series. Fig. 3 displays the computational model and the boundary conditions. The viscous damper proposed by Lysmer and Kuhlemeyer (1969) was applied at the side, top and bottom boundaries to absorb the incident motions.

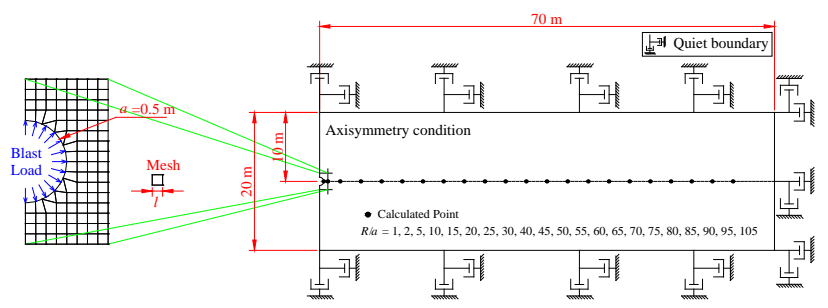

Fig. 3. Numerical model.

The size of the elements has important influence on accuracy of the numerical simulation. Kuhlemeyer and Lysmer (1973) proposed the following equation for maximum size of the mesh:

$$
\Delta l \leq \frac{c}{10 f}
$$

where $l$ is the maximum size of the elements. The results of a sensitivity study to select the optimum size of the mesh is given in the following.

The Rayleigh damping formulation, defined as follows, was used to model the small strain damping of the geologic medium:

$$
[C]=\alpha[M]+\beta[K]
$$

where $[C]$ is the damping matrix, $[M]$ is the mass matrix, $[K]$ is the stiffness matrix, $\xi$ is the damping ratio, $\alpha$ and $\beta$ are the Rayleigh coefficients that determine the frequency dependence of the damping formulation, where $\alpha$ and $\beta$ are defined as $4 \pi \xi\left(f_{1} f_{2}\right) /\left(f_{1}+f_{2}\right)$ and $\xi /[\pi$ $\left(f_{1}+f_{2}\right)$ ], respectively, and $\xi$ is the target damping ratio. The formulation matches the target damping ratio only at the frequencies of $f_{1}$ and $f_{2}$, as shown in Fig. 4. The damping of soils is known to be independent of the loading frequency, particularly in cohesionless soils or rock mass. However, the Rayleigh damping formulation is frequency-dependent and introduces numerical damping. To reduce the frequency dependence, the variation of the frequency of vibration is observed in the following. The selection process of optimum frequencies is recommended based on the calculated responses. Fig. 5 displays snapshots of PPV contours at various target damping.

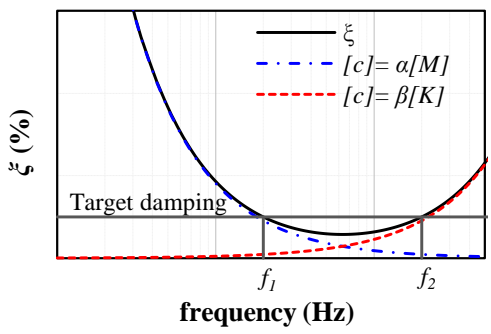

Fig. 4. Frequency dependence of Rayleigh damping formulation.

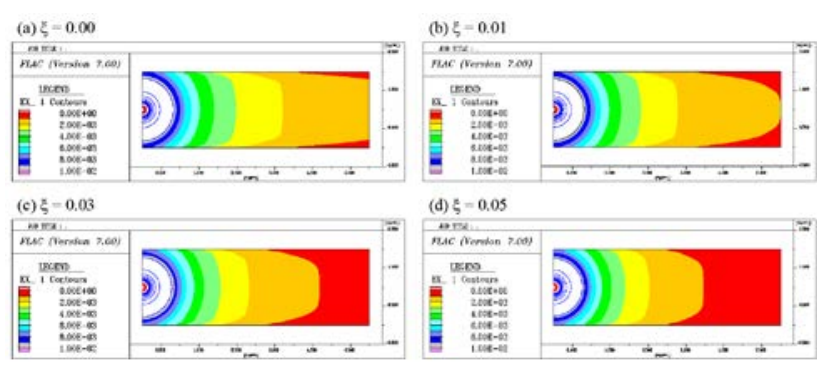

Fig. 5. Snapshots of PPV countours.

\section{VERIFCATION OF NUMERICAL MODEL}

To verify the numerical model, the results of dynamic analyses are compared to closed-form solutions. For undamped case, Duvall (1953) represents the correct solution. For damped case at far-field, the combined DFFA model represents the rigorous solution. No rigorous solution has been reported for near-field motion in damped medium. We verified the undamped model through comparisons with Duvall (1953)'s model. The damped model is verified through comparison with DFFA model.

Fig. 6 compares the attenuation curves calculated from numerical simulations and from Duvall (1953)'s equation. The computational model displayed in Fig. 3. For simulation, $n=0.25, m=0.5, v=0.25, \rho=2,500$ $\mathrm{kg} / \mathrm{m}^{3}, c=2,000 \mathrm{~m} / \mathrm{s}$, and $p_{0}=1 \mathrm{MPa}$ were applied. The predominant frequency of the impulse load used in 
Duvall (1953)'s model cannot be determined from a FFT because the due to a long tail of the loading. We used three sizes for the element mesh, which were 0.25 , $0.10,0.05 \mathrm{~m}$. Using Eq. (5), the maximum frequency that can be propagated are $800 \mathrm{~Hz}, 2,000 \mathrm{~Hz}, 4,000 \mathrm{~Hz}$ respectively.

It is shown that the numerical results in the case of the smallest mesh size are in excellent agreement with the closed-form solution, thus verifying that the model can accurately predict the impulse load propagation. For numerical simulation, the calculated response are very similar to the analytical solution, demonstrating that not only the peak value but the entire time history matches the exact solution. However, it is shown that a significantly small element size is needed for accurate estimate of the propagation.
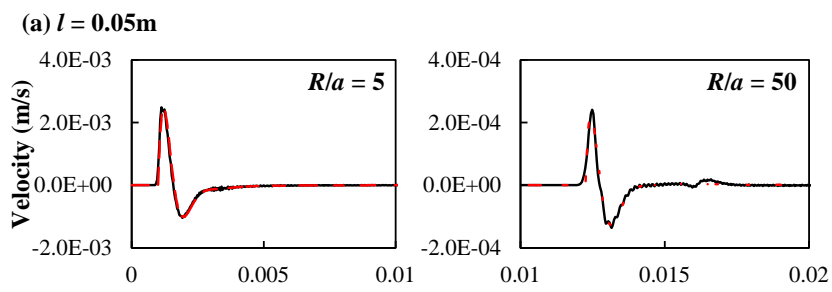

(b) $l=0.10 \mathrm{~m}$
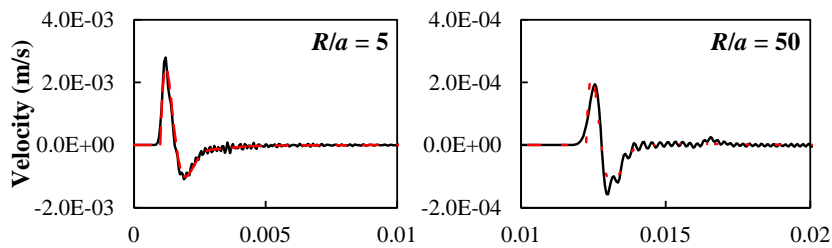

(c) $l=0.25 \mathrm{~m}$
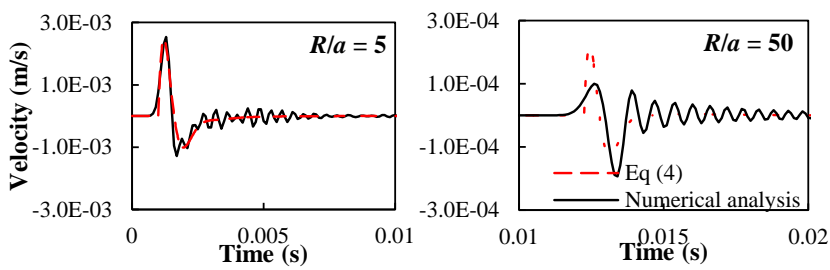

Fig. 6. Influence of mesh size on accuracy of the numerical solution.

Fig. 7 compares the far-field attenuation curve calculated from numerical simulations and DFFA. $C$ was set to $2,000 \mathrm{~m} / \mathrm{s}$ and damping ratio of $5 \%$ was used. A half-sine pulse with a frequency of $100 \mathrm{~Hz}$ was applied. The frequencies for Rayleigh damping formulation were varied, as shown in Fig. We used predominant frequency of the input motion $\left(f_{p}\right)$ for $f_{1}$ in all analyses. Various values were used for $f_{2}$, which were $2 f_{p}, 10 f_{p}$, and $\omega$, which is the angular frequency defined in Eq. (3) . The numerical solution provides a good estimate of the attenuation if $f_{p}$, and $\omega$ are selected. The amplitude of the motion is underestimated when lower values for $f_{2}$ are selected. It is because the impulse load generates pulses which are greatly higher than the predominant frequency, which are filtered when $2 f_{p}$, and $\omega$ or $10 f_{p}$, and $\omega$ are used for $f_{2}$.
The comparisons highlight that the numerical model is very accurate and reliable in predicting the attenuation of blast induced vibration. The proposed numerical model can be used in practice to perform dynamic analysis of nearby structures or to develop site-specific source-to-site attenuation relationship
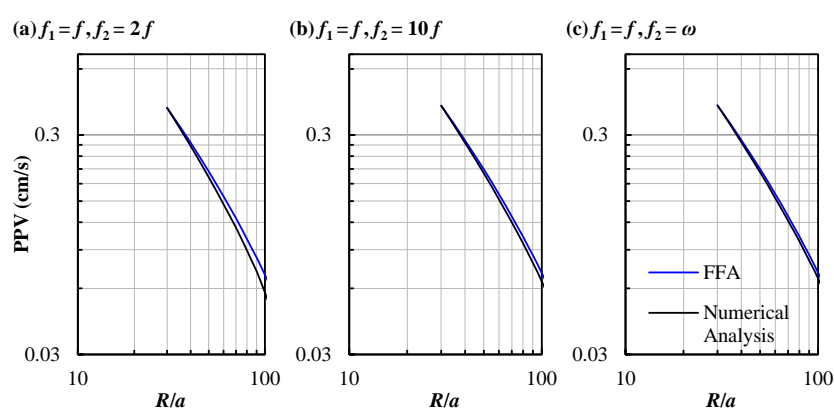

Fig. 7. Effect of selected frequencies of Rayeligh damping formulation of attenuation curves.

\section{CONCLUSIONS}

We present a numerical model to simulate blast induced vibration propagation. The solutions of two sets of analytical model were compared to the numerical model for verification. It is shown that the numerical model accurately predicts the attenuation both at near-field and far-field. The near-field vibration can be accurately captured by the model, but requires very densely meshed computation domain. The accuracy of the far-field attenuation is influenced by the frequencies selected for the Rayleigh damping formulation. We propose a procedure to select optimum frequencies for the Rayleigh damping formulation. The proposed numerical model is useful and can be used in practice to perform dynamic analysis of nearby structures or to develop site-specific source-to-site attenuation relationship.

\section{REFERENCES}

1) Dowding, C. (1984): Estimating earthquake damage from explosion testing of full-scale tunnels, Advances in tunnelling technology and subsurface use, 4(3), 113-117.

2) Dowding, C. (1996): Construction vibrations, Prentice Hall, Chapter. 4

3) Duvall, W.I. (1953): Strain-wave shapes in rock near explosions, Geophysics, 18(2), 310-323.

4) Itasca Consulting Group, Itasca (2011): Fast Lagrange Analysis of Continua, Version 7.0.

5) Kuhlemeyer, R.L. and Lysmer, J. (1973), Finite element method accuracy for wave propagation problems, Journal of Soil Mechanics \& Foundations Division. Proc. ASCE, 99(SM5), 421-427.

6) Lysmer, J. and Kuhlemeyer, R. (1969): Finite element model for infinite media, Journal of Engineering Mechanics Division. ASCE, 95, 859-877.

7) Ziolkowski, A. (1993): Determination of the signature of a dynamite source using source scaling, Part 1: Theory, Geophysics, 58(8), 1174-1182. 